\title{
HERDABILIDADE PARA OS CARACTERES CICLO VEGETATIVO E ESTATURA DE PLANTA EM AVEIA
}

\author{
HERITABILITY FOR HEADING DATE AND PLANT HEIGHT IN OAT
}

\author{
Andréa Mittelmann ${ }^{1}$ Fernando Irajá Félix de Carvalho ${ }^{2}$ José Fernandes Barbosa Neto ${ }^{3}$ \\ Adriane Leite do Amaral ${ }^{4}$ Fábio Pandini $^{5}$
}

RESUMO

\begin{abstract}
Oito populações provenientes de cruzamentos entre linhagens de aveia foram avaliadas com o objetivo de estimar a herdabilidade e verificar o efeito da seleção para estatura de planta e duração do ciclo vegetativo. Quatro panículas $F_{2}$ por cruzamento foram colhidas em 1993, cada uma delas originando uma linha de plantas espaçadas no ano seguinte. As plantas $F_{3}$ foram avaliadas individualmente e classes foram estabelecidas com base na média e desvio padrão da população. Os mesmos caracteres foram avaliados em 1995, em parcelas representando as classes selecionadas. A herdabilidade foi estimada pelo método da regressão pai x progênie. A diferença entre classes foi testada como um indicador da eficiência da seleção. As estimativas de herdabilidade variaram de -0,73 a 0,84 para estatura e de 0,25 a 1,05 para ciclo vegetativo, estando a diferenca entre classes, em geral, associada a valores elevados de herdabilidade. A herdabilidade para os caracteres estatura de planta e duração do ciclo vegetativo para algumas populações foi alta, evidenciando a viabilidade da seleção em gerações precoces. A transformação de dados em unidades de desvio padrão permitiu a redução dos efeitos da interação genótipo $x$ ambiente, levando a uma maior precisão das estimativas obtidas.
\end{abstract}

Palavras-chave: caracteres adaptativos, regressão pai $x$ progênie, Avena sativa.

\section{SUMMARY}

Eight crosses among oat lines were evaluated aiming to estimate heritability and to verify the effect of selection for plant height and heading date. Four $F_{2}$ panicles from each cross were harvested in 1993. Each one generated one line of spaced plants in the field in the following year. The $F_{3}$ plants were individually evaluated and separated in classes based on mean population and standard deviation. The same traits were evaluated in 1995 in plots representing the different classes.
Heritability was estimated by parent $x$ offspring regression method. Difference among classes was an indicator of selection efficiency. Heritability estimates varied from -0,73 to 0,84 for plant height and from 0,25 to 1,05 for heading date. Difference among classes was generally associated with high values of heritability. The heritability of the traits was high, indicating feasibility of selection in segregant generations. The conversion of data in standard deviation units allowed a reduction of genotype $x$ environment interaction, leading to more precise estimates.

Key words: adaptative traits, parent $x$ offspring regression, Avena sativa.

\section{INTRODUÇÃO}

No desenvolvimento de linhagens endogâmicas, estimativas de herdabilidade obtidas pelo coeficiente da regressão pai x progênie $\left(b_{x y}\right)$ permitem a previsão do ganho de seleção em gerações correspondentes, em populações semelhantes e ambientes semelhantes (GIBSON, 1996). Assim sendo, a informação obtida por meio dos valores estimados de herdabilidade permite ao melhorista tomar decisões quanto a métodos e estratégias de melhoramento.

Os caracteres estatura de planta e duração do ciclo vegetativo têm sido considerados importantes nos programas de melhoramento de aveia no Sul do Brasil. Plantas de estatura baixa apresentam menores problemas de acamamento e

\footnotetext{
${ }^{1}$ Engenheiro Agrônomo, Mestre, Universidade de São Paulo (USP), Escola Superior de Agricultura Luiz de Queiroz.

${ }^{2}$ Engenheiro Agrônomo, PhD., Universidade Federal de Pelotas, Faculdade de Agronomia Eliseu Maciel.

${ }^{3}$ Engenheiro Agrônomo, PhD., Universidade Federal do Rio Grande do Sul (UFRGS), Faculdade de Agronomia, Av. Bento Gonçalves, 7712, CP 776, Porto Alegre, RS, 91591-970. E-mail: jfbn@ufrgs.br. Autor para correspondência.

${ }^{4}$ Engenheiro Agrônomo, Mestre, UFRGS, Faculdade de Agronomia.

${ }^{5}$ Engenheiro Agrônomo, Mestre, USP, Escola Superior de Agricultura Luiz de Queiroz. 
suportam o uso de doses mais elevadas de fertilizantes. Da mesma forma, a duração do ciclo da cultura é outro caráter de importância adaptativa. Genótipos precoces são desejáveis por permitirem a sucessão com os cultivos de verão. De maneira geral, os valores de herdabilidade estimados para estes caracteres em cereais de estação fria têm variado de magnitude média a alta. Em trigo, CARVALHO et al. (1981) obtiveram valores entre 0,53 e 0,93 para estatura de planta. Em aveia, foram obtidas estimativas de herdabilidade para estatura de 0,43 a 0,92 e de 0,19 a 0,59 para ciclo (AMARAL $\boldsymbol{e t}$ al., 1996). Outros autores apresentaram valores de 0,61 e 0,87 para estatura (PETR \& FREY, 1966) e de 0,56 e 0,60 para ciclo (JONES \& FREY, 1960).

Este trabalho teve por objetivo estimar a herdabilidade para os caracteres agronômicos estatura de planta e duração do ciclo vegetativo em quatorze populações de aveia, verificando o efeito da seleção para estes caracteres em gerações precoces.

\section{MATERIAL E MÉTODOS}

O trabalho foi desenvolvido a campo na Estação Experimental Agronômica da Universidade Federal do Rio Grande do Sul, em Eldorado do Sul, nos anos de 1993 a 1995.

Inicialmente, em 1993, foram selecionadas quatro panículas $\mathrm{F}_{2}$ resultantes de cruzamento entre linhagens de aveia. Em 1994, cada panícula colhida originou uma linha de plantas com $3 \mathrm{~m}$ de comprimento. As plantas $\mathrm{F}_{3}$, semeadas com espaçamento de $30 \mathrm{~cm}$ na linha, foram avaliadas individualmente para os caracteres estatura e duração do ciclo vegetativo. As medidas de estatura foram tomadas 21 dias após a antese, correspondendo à distância do solo até a extremidade superior da panícula principal. O ciclo vegetativo, compreendendo o período da emergência até a exposição total da panícula principal, foi determinado semanalmente. Dentro de cada cruzamento, foram estabelecidas classes, com base na média do cruzamento e no desvio padrão, considerando os dois caracteres avaliados. Algumas plantas, representando as diferentes classes, foram colhidas, e sementes de plantas pertencentes à mesma classe foram misturadas. Foram consideradas intermediárias, para ambos os caracteres, plantas com avaliações dentro do intervalo de um desvio padrão acima e um abaixo da média da população. Entre um e dois desvios acima da média foram consideradas altas e tardias. Foram incluídas como baixas ou precoces as plantas com observações de um a dois desvios abaixo da média para estatura e ciclo, respectivamente. As plantas selecionadas que apresentavam dois desvios abaixo da média da população para estatura foram identificadas como muito baixas.

Em 1995, foi estabelecido um ensaio em blocos casualizados com quatro repetições, compreendendo oito populações $F_{4}$. As parcelas, representando as classes estabelecidas dentro de cada cruzamento, foram constituídas por uma linha de $3 \mathrm{~m}$ de comprimento com densidade de 50 sementes por metro linear. Para cada parcela, foram avaliados os caracteres estatura (média de três medidas na linha) e duração do ciclo vegetativo (número de dias para que $80 \%$ das plantas da linha emitissem a primeira panícula). Com base nos dados de 1995 para a geração $F_{4}$, foi realizado o teste $t$ de comparação de médias entre classes dentro de cada cruzamento para os dois caracteres em estudo.

As estimativas de herdabilidade foram obtidas pelo método da regressão pai x progênie proposto por LUSH (1940), com base nos dados das gerações $\mathrm{F}_{3}$ e $\mathrm{F}_{4}$. Cada população foi analisada individualmente. Os valores observados foram transformados em unidades de desvio padrão conforme FREY \& HORNER (1957) e submetidos novamente à analise de regressão. A significância dos coeficientes de regressão obtidos foi avaliada por teste $\mathrm{F}$ em nível de 5\% de probabilidade de erro.

\section{RESULTADOS E DISCUSSÃO}

As oito populações derivadas de cruzamentos de diferentes linhagens de aveia possibilitaram cinco valores significativos de herdabilidade para o caráter estatura de planta e seis para o caráter ciclo vegetativo. Os valores de herdabilidade (b) estimados variaram de $-0,54$ a 1,28 para estatura e de 0,37 a 1,21 para ciclo (Tabela 1). A média geral do experimento para estatura foi de $100,2 \mathrm{~cm}$ em 1994 e 121,1cm em 1995. Para o caráter ciclo, a média geral foi de 104,7 dias em 1994 e 90,3 dias em 1995, ocorrendo variação acentuada entre anos para os dois caracteres.

Pela conversão dos valores observados em unidades de desvio padrão, as estimativas de herdabilidade obtidas tiveram uma amplitude de 0,73 a 0,84 para estatura e de 0,25 a 1,05 para ciclo (Tabela 1). Para algumas populações, a transformação dos dados trouxe acréscimo nos valores de herdabilidade; entretanto, em outras houve decréscimo. No âmbito geral, não foi eliminada a ocorrência de valores acima da unidade e negativos, o que indica possivelmente a influência de outros fatores que não apenas a interação genótipo $\mathrm{x}$ ambiente. 
Tabela 1 - Estimativas de herdabilidade dos caracteres estatura de planta e ciclo vegetativo obtidas a partir dos dados originais (b) e após transformação em unidades de desvio padrão (b'). EEA-UFRGS, 1994/95.

\begin{tabular}{lccccc}
\hline \multirow{2}{*}{ Cruzamento } & \multicolumn{2}{c}{ Estatura de planta } & \multicolumn{2}{c}{ Ciclo vegetativo } \\
\cline { 2 - 5 } & $\mathrm{b}$ & $\mathrm{b}^{\prime}$ & $\mathrm{b}$ & $\mathrm{b}^{\prime}$ \\
\hline UFRGS 881920/UFRGS 7 & 0,57 & 0,72 & 0,82 & 0,71 \\
UFRGS 15/UFRGS 14 & - & - & 1,21 & 1,05 \\
UFRGS 881920/UFRGS 14 & 1,28 & 0,65 & 0,37 & 0,25 \\
UFRGS 881920/UFRGS 15 & 0,70 & 0,67 & 0,70 & 0,96 \\
UFRGS 884077/UFRGS 14 & - & - & 0,79 & 1,05 \\
UFRGS 881920/UFRGS 884070-2 & $-0,54$ & $-0,73$ & - & - \\
UFRGS 884077//UFRGS 8/UFRGS 884095 & 0,47 & 0,84 & - & - \\
UFRGS 884068/IA 76Q337 & - & - & 0,94 & 0,99 \\
\hline
\end{tabular}

IA introdução australiana

Os valores de herdabilidade (b') para o caráter estatura de planta foram médios a altos, com exceção do cruzamento UFRGS 881920 x UFRGS $884070-2$, indicando a viabilidade de obter ganhos com a seleção em gerações precoces. Com relação à duração do ciclo vegetativo, os valores de b' foram altos, em geral mais elevados do que para estatura, havendo apenas um valor considerado baixo, correspondente ao cruzamento UFRGS 881920 x UFRGS 14.

A persistência de valores superiores à unidade, verificados neste trabalho, pode ser explicada pela diferença de critérios adotados para a determinação do caráter duração do ciclo vegetativo nos dois anos do experimento. A diferença de densidade de semeadura entre anos pode ter afetado as estimativas de herdabilidade obtidas antes da conversão já que foram utilizadas plantas espaçadas nos dois primeiros anos e linha cheia no último. Segundo GEADELMANN \& FREY (1975) um dos fatores de grande influência na interação genótipo $\mathrm{x}$ ambiente é a competição entre os genótipos diferenciados existentes em uma população, que assume maior importância quanto menor o espaçamento entre plantas.

A drástica redução de variabilidade entre as médias das progênies em gerações avançadas determina decréscimos em valores estimados de herdabilidade (CARVALHO et $\boldsymbol{a l} ., 1981)$. Uma correção das estimativas de herdabilidade com base no coeficiente de endogamia foi (cm). EEA-UFRGS, 1994/95. proposta por NYQUIST (1991). Neste experimento, as estimativas de herdabilidade foram obtidas por meio da regressão entre genitores $\mathrm{F}_{3}$ e progênies $\mathrm{F}_{4}$, porém segundo o modelo proposto, não são necessários ajustes pois foram utilizadas famílias $\mathrm{F}_{3}$ derivadas de plantas $\mathrm{F}_{2}$ (GIBSON, 1996).

A manutenção da diferença entre classes selecionadas em uma geração em relação à subseqüente pode ser considerada como indicadora da eficiência da seleção. A diferença significativa entre classes para estatura (Tabela 2) e ciclo (Tabela 3 ) esteve, de modo geral, associada a valores médios a altos de herdabilidade, evidenciando que as diferenças selecionadas foram influenciadas por fatores genéticos herdáveis. As diferenças entre os genitores determinam a variabilidade genética existente nas populações. Segundo FALCONER (1960) a resposta à seleção pode ser otimizada, através da herdabilidade, devido à maior diferença entre os pais.

Em alguns casos, os valores de herdabilidade não foram suficientes para explicar a manutenção das diferenças entre seleções, como no caso da população UFRGS 881920 x UFRGS 14 em relação ao ciclo vegetativo. Nesse caso, é possível inferir que a interação genótipo $x$ ambiente contribuiu de maneira favorável para a manutenção da diferença entre classes. $\mathrm{O}$ fato de a herdabilidade estimada para esta população antes da conversão em unidades de desvio padrão (b) possuir valor mais elevado parece corroborar esta hipótese.

Tabela 2 - Herdabilidade (b') e média das diferentes classes para o caráter estatura de planta

\begin{tabular}{|c|c|c|c|}
\hline Cruzamento & Herdabilidade & Classe & Média $(\mathrm{cm})$ \\
\hline UFRGS 881920/UFRGS 7 & 0,72 & $\begin{array}{c}\text { Alta } \\
\text { Intermediária } \\
\text { Baixa }\end{array}$ & $\begin{array}{l}134,5 \mathrm{a}^{1} \\
130,2 \mathrm{a} \\
125,2 \mathrm{~b}\end{array}$ \\
\hline UFRGS 881920/UFRGS 14 & 0,65 & $\begin{array}{c}\text { Intermediária } \\
\text { Baixa }\end{array}$ & $\begin{array}{l}118,1 \mathrm{a} \\
111,3 \mathrm{~b}\end{array}$ \\
\hline UFRGS 881920/UFRGS 15 & 0,67 & $\begin{array}{c}\text { Intermediária } \\
\text { Baixa }\end{array}$ & $\begin{array}{l}120,2 \mathrm{a} \\
116,6 \mathrm{~b}\end{array}$ \\
\hline UFRGS 881920/UFRGS 884070-2 & $-0,73$ & $\begin{array}{c}\text { Alta } \\
\text { Intermediária } \\
\text { Baixa }\end{array}$ & $\begin{array}{l}112,7 \quad \mathrm{c} \\
117,0 \mathrm{~b} \\
120,3 \mathrm{a}\end{array}$ \\
\hline UFRGS 884077//UFRGS 8/UFRGS 884095 & 0,83 & $\begin{array}{c}\text { Baixa } \\
\text { Muito baixa }\end{array}$ & $\begin{array}{l}143,1 \mathrm{a} \\
124,3 \mathrm{~b}\end{array}$ \\
\hline
\end{tabular}

1 Valores não seguidos de mesma letra, dentro de um mesmo cruzamento, diferem significativamente pelo teste t a $5 \%$ de probabilidade.

Ciência Rural, v. 31, n. 6, 2001. 
Tabela 3 - Herdabilidade (b') e média das diferentes classes para o caráter estatura de planta (cm). EEA-UFRGS, 1994/95.

\begin{tabular}{|c|c|c|c|}
\hline Cruzamento & Herdabilidade & Classe & Média (dias) \\
\hline UFRGS 881920/UFRGS 7 & 0,71 & $\begin{array}{c}\text { Intermediária } \\
\text { Intermediária } \\
\text { Precoce }\end{array}$ & $\begin{array}{l}90,5 \mathrm{a}^{1} \\
90,5 \mathrm{a} \\
80,5 \mathrm{~b}\end{array}$ \\
\hline UFRGS 15/UFRGS 14 & 1,05 & $\begin{array}{c}\text { Tardia } \\
\text { Intermediária }\end{array}$ & $\begin{array}{c}101,0 \mathrm{a} \\
86,8 \mathrm{~b}\end{array}$ \\
\hline UFRGS 881920/UFRGS 14 & 0,25 & $\begin{array}{c}\text { Intermediária } \\
\text { Precoce }\end{array}$ & $\begin{array}{l}98,3 \mathrm{a} \\
95,7 \mathrm{~b}\end{array}$ \\
\hline UFRGS 881920/UFRGS 15 & 0,96 & $\begin{array}{l}\text { Intermediária } \\
\text { Intermediária }\end{array}$ & $\begin{array}{l}105,0 \mathrm{a} \\
101,1 \mathrm{~b}\end{array}$ \\
\hline UFRGS 884077/UFRGS 14 & 1,05 & $\begin{array}{c}\text { Tardia } \\
\text { Intermediária } \\
\text { Intermediária } \\
\text { Precoce }\end{array}$ & $\begin{array}{l}79,0 \mathrm{~b} \\
89,2 \mathrm{a} \\
77,5 \mathrm{~b} \\
72,0 \mathrm{c}\end{array}$ \\
\hline UFRGS 884068/IA 76Q337 & 0,99 & $\begin{array}{c}\text { Tardia } \\
\text { Intermediária }\end{array}$ & $\begin{array}{l}96,0 \mathrm{a} \\
82,7 \mathrm{~b}\end{array}$ \\
\hline
\end{tabular}

1 Valores não seguidos de mesma letra, dentro de um mesmo cruzamento, diferem significativamente pelo teste $\mathrm{t}$ a $5 \%$ de probabilidade. IA introdução australiana

É importante destacar que os caracteres ciclo vegetativo e estatura de planta possuem distribuição contínua e que o estabelecimento de classes tende a provocar algumas distorções. Assim, para o caráter estatura de planta, a população UFRGS 881920 x UFRGS 7 não apresentou diferença significativa entre as classes alta e intermediária, embora possuísse herdabilidade de média a alta. Isso ocorreu possivelmente devido à utilização de um intervalo muito amplo na seleção. Decorrente disso, a diferença para o caráter entre plantas da mesma classe fosse maior do que a encontrada entre plantas consideradas de classes diferentes.

O critério de agrupamento das plantas colhidas em 1994 foi baseado em ambos os caracteres avaliados. Por isso, para alguns cruzamentos, ocorreram dois tratamentos com a mesma classificação em relação ao ciclo vegetativo. Da mesma forma que o observado para estatura, o estabelecimento de classes com amplitude elevada fez com que na população UFRGS 881920 x UFRGS 15 houvesse diferença significativa entre classes consideradas semelhantes para ciclo em 1994.

Além do intervalo entre classes e da interação genótipo $\mathrm{x}$ ambiente, o pequeno número de progênies por cruzamento, resultando em um número reduzido de pontos na análise de regressão pode ter contribuído para estes resultados.

\section{CONCLUSÕES}

A herdabilidade para os caracteres estatura de planta e duração do ciclo vegetativo foi alta, viabilizando a seleção eficaz em gerações precoces de autofecundação.

O uso da transformação de dados para a supressão dos efeitos da interação genótipo $\mathrm{x}$ ambiente permitiu uma melhor interpretação das estimativas obtidas através da regressão pai x progênie.

\section{REFERÊNCIAS BIBLIOGRÁFICAS}

AMARAL, A., CARVALHO, F.I.F., FEDERIZZI, L.C., $\boldsymbol{e}$ t al. Estimativa da herdabilidade para os caracteres adaptativos ciclo e estatura em aveia. Ciência Rural, Santa Maria, v.26, p.33-37, 1996.

CARVALHO, F.I.F., UITDEWILLIGEN, W.P.M., FEDERIZZI, L.C.O., et al. Herdabilidade do caráter estatura de planta em trigo: estimativa através do coeficiente de regressão. Pesquisa Agropecuária Brasileira, Brasília, v.16, v.55-67, 1981.

FALCONER, D.S. Introduction to quantitative genetics. 3 ed. Harlow (UK) : Longman Scientific and Technical, 1987. $279 \mathrm{p}$.

FREY, K.J., HORNER, T. Heritability in standard units. Agronomy Journal, Madison, v.49, p.59-62, 1957.

GEADELMANN, J.L., FREY, K.J. Direct and indirect mass selection for grain yield in bulk oat populations. Crop Science, Madison, v.15, p.490-494, 1975.

GIBSON, P.T. Correcting for inbreeding in parent-offspring regression estimates of heritability with non-additive and genotype X environment effects present. Crop Science, Madison, v.36, p.594-600, 1996.

JONES, K.R., FREY, K.J. Heritability percentages and degrees of dominance for quantitative characters in oats. St J Science, v.35, p.49-58, 1960.

LUSH, J.L. Intrasire correlations on regressions of offspring on dam as a method of estimating heritability ofcharacteristics. Proc Amer Soc An Prod, p.293-301, 1940.

NYQUIST, W.E. Estimation of heritability and prediction of selection response in plant populations. Critical Reviews in Plant Science, v.10, p.235-322, 1991

PETR, F.C., FREY, K.J. Genotipic correlations, dominance, and heritability of quantitative characters in oats. Crop Science, Madison, v.6, p.259-262, 1966 